\title{
NOTES ON SRAFFA'S FIXED CAPITAL MODEL
}

\author{
J. E. WOODS ${ }^{1}$
}

(Received 22 February 1982; revised 19 September 1983)

\begin{abstract}
This paper considers a single-product industries, fixed capital model discussed briefly by Sraffa in Chapter $X$ of his book. The analysis is elementary, being based on the Perron-Frobenius Theorem on semipositive indecomposable matrices and the direct calculation of a certain matrix inverse. This generalises the approach adopted for circulating capital models. It also demonstrates that common economic and mathematical frameworks exist for both circulating and fixed capital models.
\end{abstract}

\section{Introduction}

\subsection{Introductory remarks}

The purpose of this paper is to provide an elementary formal account of a particular fixed capital model discussed by Sraffa in Chapter X of [8]. It is well known by now, following the work of von Neumann [4] and Sraffa, that a proper treatment of fixed capital requires a joint production model; the treatment of depreciation by means of a priori specified coefficients, characteristic of the Dynamic Leontief and related multisectoral models (see, for example, Chapter 5 of Woods [9], Chapter VI of Morishima [2] or Chapter 8 of Burmeister and Dobell [1]), ignores the economic, as opposed to the technical, dimension of the problem. Indeed, according to Sraffa, the rationale for studying joint production models is the provision of an adequate framework for the analysis of fixed capital: "The interest of Joint Products does not lie so much in the familiar examples of wool and mutton, or wheat and straw, as in its being the genus of which Fixed Capital is the leading species." ([8], page 63).

The particular feature of the model in Chapter $X$ of [8] is that joint production arises solely from the presence of fixed capital. "We shall regard durable

\footnotetext{
${ }^{1}$ Department of Economics, University of Dublin, Trinity College, Dublin 2, Ireland.

(c) Copyright Australian Mathematical Society 1984, Serial-fee code 0334-2700/84
} 
instruments of production as part of the annual intake of a process, on the same footing as such means of production (e.g. raw materials) as are entirely used up in the course of a year; while what is left of them at the end of the year will be treated as a portion of the annual joint product of the industry, ofwhich the more conspicuous part consists of the marketable commodity that is the object of the process. For example, a knitting machine enters the means of production at the beginning of the year, along with the yarn, the fuel, etc., with which it is employed; and at the end of the year the partly worn-out, older machine which emerges from the process will be regarded as a joint product with the year's output of stockings." ([8], page 63).

We consider a closed economy of $(n+1)$ sectors, with only one sector, say the $(n+1)$ th for ease of notation, employing fixed capital. Each sector produces only one marketable commodity. A model based on these two assumptions resembles, on a strict interpretation, that presented in Sections 73-76 of [8], where it is the $g$ th sector that employs fixed capital. The two assumptions can be rationalised in the following ways: first, that a commodity produced by a sector other than the $(n+1)$ th is used as fixed capital only by the $(n+1)$ th; second, that such a commodity is used as fixed capital by the $(n+1)$ th sector and as circulating capital by at least one other sector. The resulting model is a hybrid of the single-product industries, circulating capital model of Part I of [8] and the multiple-product industries, fixed capital model of Part II of [8], especially Chapters VII-IX; it can thus be described as a single-product industries, fixed capital model.

\subsection{Summary of the paper}

As stated at the outset, the main purpose of this paper is to construct and analyse a model that permits a proper treatment of fixed capital. A subsidiary purpose is to demonstrate that such a properly specified fixed capital model is based on the same economic principles as a circulating capital model and that the well known mathematical theory developed for the latter can also be applied to the former.

A complete description of the fixed capital model is given in Section 2.1. In Section 2.2, there is a brief review of the circulating capital model, emphasising the importance of a productivity condition in deriving the existence of a positive maximum rate of profit. Then in Section 2.3 , it is shown that an analogous productivity condition performs the same role for the fixed capital model. However, there are problems peculiar to fixed capital models; an obvious example is the determination of the optimal economic lifetime of a machine, a problem with no counterpart in the theory of circulating capital. Consequently, it might be thought that new methods are required for the analysis of fixed, as opposed to circulating, capital models. In Sections $2.2-2.7$, it is shown that an approach 
based on the direct calculation of matrix inverses suffices for both types of capital models; such an approach requires for fixed capital the derivation of new matrix results, which are given in Sections 2.4 and 2.6.

The analysis of the single-product industries, fixed capital model can thus be described as elementary, in the sense that it concentrates on the evaluation of a matrix inverse. This point does not seem to have been appreciated by other contributors to the literature on fixed capital; in this context, see Pasinetti [7].

It is clear from the brief summary in Section 1.1 that the particular fixed capital model analysed in this paper is not the most general one available (a more general single-product industries, fixed capital model is discussed in Woods [10]). This simple model has been chosen so that the underlying principles can be perceived. In Section 3, we demonstrate that the framework can be extended to provide an analysis of more general fixed capital models.

\section{A single-product industries, fixed capital model}

\subsection{Description of the fixed capital model}

The treatment of fixed capital in this paper is based on the foundations provided by von Neumann and Sraffa (see also Chapter VI of Morishima [3]). This von Neumann-Sraffa point of view implies that the same machine, at different ages, should be treated as so many different products, each with its own price. In order to determine these prices, an equal number of additional equations (and therefore of processes) is required. Accordingly, an industry which employs a durable instrument of production must be regarded as being subdivided into as many separate processes as are the total years of life of the instrument in question. Each of these processes is distinguished by the fact that it uses an instrument of a different age; and each of them 'produces', jointly with a quantity of a marketable commodity, an instrument a year older than the one which it uses - with the exception of the process using the expiring instrument in its last year, which produces singly the marketable commodity." ([8], pages 63-64).

As mentioned in Section 1.1, it is assumed that only sector $(n+1)$ employs fixed capital, with sectors $1, \ldots, n$ employing only circulating capital. We assume that each of sectors $1, \ldots, n$ has available only one production process and that there is a common period of production, say a year. We also assume that the fixed capital good employed in the $(n+1)$ th sector has a maximum physical lifetime of $N$ production periods. In the von Neumann-Sraffa approach, it is necessary to distinguish between machines of different ages, even though they are of the same type and are employed in the same sector; clearly, in the fixed capital model of this paper, there are $(N-1)$ ageing machines to be distinguished. As each sector is assumed to produce only one marketable commodity, there are $(n+1)$ 
marketable commodities to be distinguished. So, there is a total of $(n+1)+(N$ $-1)=(n+N)$ commodities in the model. The adoption of the von NeumannSraffa approach to fixed capital implies that the list of distinct commodities should be extended from the $(n+1)$ marketable commodities to $(n+N)$ to include the $(N-1)$ ageing machines. As an expository device, it may be useful to think of the latter as essentially nonmarketable, a view supported by Sraffa: "Nor is it necessary that the instruments belonging to successive age-groups should actually be marketed for their prices to be effective; since even though these are only bookvalues, they are the basis for correctly allocating the profits and making allowance for depreciation in the case of each age-group." ([8], page 64). We adopt this device of treating ageing machines as nonmarketable.

Having enumerated the number of distinct commodities, we can now describe production processes in more detail. In sector $j$, circulating capital inputs (i.e. marketable commodities) are combined with labour to yield a quantity of the $j$ th marketable commodity, where $j=1, \ldots, n$. Now consider sector $(n+1)$. As it employs a durable instrument of production, this sector (in contrast to every other) must have more than one production process. In the first process available to sector $(n+1)$, a new machine is combined with circulating capital and labour inputs to produce a quantity of marketable commodity $(n+1)$ and a one-year old machine. This one-year old machine can then enter another process with circulating capital and labour inputs to yield another quantity of commodity $(n+1)$ and a two-year old machine-this is the second process available to sector $(n+1)$. This two-year old machine can then enter a third process and so on. As the machine has a maximum physical lifetime of $N$ production periods, there is clearly a total of $N$ processes available to sector $(n+1)$, assuming that each process lasts for one year, as in sectors $1, \ldots, n$, and that each process employs only one ageing machine. So, the total number of processes in the economy is $(n+N)$; there is equality between the number of processes and the number of commodities.

The commodities are numbered in the following way:

$$
\begin{array}{ll}
1, \ldots, n+1 & \text { the marketable commodities, in- } \\
& \text { cluding the new machine em- } \\
& \text { ployed by sector }(n+1) \\
& \text { the }(v-1) \text {-year old machine em- } \\
& \text { ployed as input into the } v \text { th pro- } \\
& \text { cess of sector }(n+1), v= \\
& 2, \ldots, N .
\end{array}
$$

As we distinguish between marketable commodity prices and ageing machine prices, we partition the price vector $p=\left(p_{1}, \ldots, p_{n+1} ; p_{n+2}, \ldots, p_{n+N}\right)$ into

$p^{f}=\left(p_{1}, \ldots, p_{n+1}\right)$, the subvector of marketable, or final, commodity prices; 
and

$p^{m}=\left(p_{n+2}, \ldots, p_{n+N}\right)$, the subvector of ageing machine prices.

We now define the production conditions in each sector. As there are $(n+N)$ commodities that can be used as inputs, as (homogeneous) labour is assumed to be indispensable in production, and as each sector produces only one marketable commodity, it follows that we can describe any production process in sectors $1, \ldots, n$ by an $(n+N+2)$ tuple. The first $(n+1)$ components of this activity vector refer to marketable commodity inputs, the next $(N-1)$ components refer to ageing machine inputs, the $(n+N+1)$ th component refers to the labour input and the last component to the marketable commodity output of the process. We assume constant returns to scale in all production processes. First, consider sector $j$, where $j=1, \ldots, n: a_{i j}$ is the input of marketable commodity $i$ per unit of output of marketable commodity $j(i=1, \ldots, n+1 ; j=1, \ldots, n)$ and

$$
A^{i}=\left[\begin{array}{c}
a_{i j} \\
\vdots \\
a_{n+1, j}
\end{array}\right]
$$

is the subvector of marketable commodity input-output coefficients for sector $j$. As sector $j$ does not employ fixed capital inputs, define;

$\mathbf{0}_{N-1}=$ the subvector of ageing machine inputs for sector $j$,

$l_{j}=$ input of labour per unit of output of marketable commodity $j$, and

$b_{j}=$ output of marketable commodity $j$.

Using the constant returns to scale assumption, we can describe the production process available to sector $j$ by the $(n+N+2)$ tuple,

$$
\left[\begin{array}{c}
A^{j} \\
\mathbf{0}_{N-1} \\
l_{j} \\
\hdashline 1
\end{array}\right]
$$

REMARK 1. We choose the obvious normalisation (i.e. output) for the $j$ th sector's activity vector.

Now consider the production conditions in sector $(n+1)$. As remarked above, there are $N$ processes to be described. Let $a_{i, n+v}$ be the input of marketable commodity $i$ into the $v$ th process $(i=1, \ldots, n+1 ; v=1, \ldots, N)$ and

$$
A^{n+v}=\left[\begin{array}{c}
a_{1, n+v} \\
\vdots \\
a_{n+1, n+v}
\end{array}\right]
$$

is the subvector of marketable commodity inputs into the $v$ th process. There is one ageing machine input into each process, except the first, in sector $(n+1)$. Let 
$M^{v}=e^{v}$, the $v$ th unit vector with $(N-1)$ components, be the vector of ageing machine inputs into the $(v+1)$ th process $(v=1, \ldots, N-1), 0_{N-1}$ be the vector of ageing machine inputs into the first process, $l_{n+v}$ be the labour input into the $v$ th process $(v=1, \ldots, N)$, and $b_{n+v}$ be the output of marketable commodity $(n+1)$ from the $v$ th process $(v=1, \ldots, N)$. We stack the commodity input subvectors, the labour input and commodity output so that we can describe the first process in sector $(n+1)$ by

$$
\left[\begin{array}{l}
A^{n+1} \\
\mathbf{0}_{N-1} \\
l_{n+1} \\
\hdashline b_{n+1}
\end{array}\right]
$$

and the $(v+1)$ th process by

$$
\left[\begin{array}{c}
A^{n+v+1} \\
M^{v} \\
l_{n+v+1} \\
\hdashline b_{n+v+1}
\end{array}\right], \quad v=1, \ldots, N-1 .
$$

REMARK 2. Without loss of generality, we can normalise a production process in sector $(n+1)$ so that there is unit input of an ageing machine, not a unit output of a marketable commodity.

Finally, let $w$ be the uniform wage rate and $r$ be the uniform rate of profit.

We can now write down the price equations for the economy. First, the price equation for sector $j, j=1, \ldots, n$,

$$
(1+r)\left[p^{f} A^{j}+p^{m} \mathbf{0}\right]+w l_{j}=p_{j}
$$

where $p^{f} A^{j}$ is the value of (circulating) capital advanced per unit of output in sector $j, r p^{f} A^{j}$ is the profit on this capital and $w l_{j}$ is the wage per unit of output. For sector $(n+1)$, there are $N$ price equations, one for each process;

$$
\begin{aligned}
& (1+r)\left[p^{f} A^{n+1}+p^{m} 0\right]+w l_{n+1}=p_{n+1} b_{n+1}+p^{m} M^{1}, \\
& (1+r)\left[p^{f} A^{n+2}+p^{m} M^{1}\right]+w l_{n+2}=p_{n+1} b_{n+2}+p^{m} M^{2} \text {, } \\
& (1+r)\left[p^{f} A^{n+v+1}+p^{m} M^{v}\right]+w l_{n+v+1}=p_{n+1} b_{n+v+1}+p^{m} M^{v+1}, \\
& (1+r)\left[p^{f} A^{n+N}+p^{m} M^{N-1}\right]+w l_{n+N}=p_{n+1} b_{n+N}+p^{m} \mathbf{0},
\end{aligned}
$$

where 0 on the right-hand side of the last equation denotes the fact that there are no ageing machine outputs from the last process, just as $\mathbf{0}$ on the left-hand side of the first equation denotes the fact that there are no ageing machine inputs into the first process. Note that $M^{v}$, the ageing machine input vector into the $(v+1)$ th process, is also the ageing machine output vector from the $v$ th process. 
Combine (1) and (2) to obtain

$$
\begin{aligned}
& (1+r)\left[p^{f} p^{m}\right]\left[\begin{array}{ccccc}
A^{1} & \ldots & A^{j} & \ldots & A^{n} \\
\mathbf{0} & \ldots & \mathbf{0} & \ldots & \mathbf{0}
\end{array} \mid\right. \\
& \left.\begin{array}{ccccccc}
A^{n+1} & A^{n+2} & \ldots & A^{n+v+1} & \ldots & A^{n+N-1} & A^{n+N} \\
\mathbf{0} & M^{1} & \ldots & M^{v} & \ldots & M^{N-2} & M^{N-1}
\end{array}\right] \\
& +w\left[l_{1} \ldots l_{j} \ldots l_{n} \mid l_{n+1} l_{n+2} \ldots l_{n+v+1} \ldots l_{n+N-1} l_{n+N}\right] \\
& =\left[p^{f} p^{m}\right]\left[\begin{array}{ccccc}
e^{1} & \ldots & e^{j} & \ldots & e^{n} \\
\mathbf{0} & \ldots & \mathbf{0} & \ldots & \mathbf{0}
\end{array} \mid\right. \\
& \left.\begin{array}{ccccccc}
\underline{e}^{n+1} & \underline{e}^{n+2} & \ldots & \underline{e}^{n+v+1} & \ldots & \underline{e}^{n+N-1} & \underline{e}^{n+N} \\
M^{1} & M^{2} & \ldots & M^{v+1} & \ldots & M^{N-1} & 0
\end{array}\right] .
\end{aligned}
$$

In (3a), $e^{j}$ is the $j$ th unit vector of order $(n+1), j=1, \ldots, n+1$, and $\underline{e}^{n+v}=$ $b_{n+v} e^{n+1}, v=1, \ldots, N$. Let,

$$
\begin{aligned}
& A_{N}=\left[\begin{array}{ccccc}
A^{1} & \ldots & A^{j} & \ldots & A^{n} \\
\mathbf{0} & \ldots & \mathbf{0} & \ldots & \mathbf{0}
\end{array} \mid\right. \\
& \left.\begin{array}{ccccccc}
A^{n+1} & A^{n+2} & \ldots & A^{n+v+1} & \ldots & A^{n+N-1} & A^{n+N} \\
\mathbf{0} & M^{1} & \ldots & M^{v} & \ldots & M^{N-2} & M^{N-1}
\end{array}\right], \\
& l_{N}=\left[l_{1} \ldots l_{j} \ldots l_{n} \mid l_{n+1} l_{n+2} \ldots l_{n+v+1} \ldots l_{n+N-1} l_{n+N}\right] \text {, } \\
& \boldsymbol{B}_{N}=\left[\begin{array}{ccccc}
e^{1} & \ldots & e^{j} & \ldots & e^{n} \\
\mathbf{0} & \ldots & \mathbf{0} & \ldots & \mathbf{0}
\end{array} \mid\right. \\
& \left.\begin{array}{ccccccc}
\underline{e}^{n+1} & \underline{e}^{n+2} & \ldots & \underline{e}^{n+v+1} & \ldots & \underline{e}^{n+N-1} & \underline{e}^{n+N} \\
M^{1} & M^{2} & \ldots & M^{v+1} & \ldots & M^{N-1} & 0
\end{array}\right] .
\end{aligned}
$$

The triple $\left\{A_{n}, l_{N} ; B_{N}\right\}$ defines technique $(N)$ in which sector $j$ employs only circulating capital, $j=1, \ldots, n$, and sector $(n+1)$ employs its ageing machine for $N$ periods. We amend the previous notation by inserting the subscript " $N$ " into the price vector, $p$, and the wage rate, $w$. Equation (3a) can then be rewritten as

$$
(1+r) p_{N} A_{N}+w_{N} l_{N}=p_{N} B_{N},
$$

or

$$
p_{N}\left[B_{N}-(1+r) A_{N}\right]=w_{N} l_{N}
$$

or

$$
p_{N}=w_{N} l_{N}\left[B_{n}-(1+r) A_{N}\right]^{-1},
$$

assuming that the inverse exists.

With the derivation of (3a), the description of the fixed capital model is complete. Before analysing (3c) and (3d), the subject of Sections 2.2-2.8, there is one point worth considering. In a properly specified fixed capital model, after von 
Neumann and Sraffa, both types of capital exhibit the property of circulating capital that it lasts for only one production period. This is true trivially of circulating capital in the model outlined above. It is also true of fixed capital: a new machine enters the first process of sector $(n+1)$ and does not emerge as an output, or rather it does emerge as something different, a one-year old machine; similarly, for older machines, except for the $(N-1)$-year old machine which is completely used up in the $N$ th process. Thus each capital good, whether circulating capital or an ageing machine, last for one period in its own form. Given that a proper treatment of fixed capital is based on the same economic principles as circulating capital, it is reasonable to ask if the mathematical theory applied to solve the latter can also be used to solve the former. Hence, before analysing the fixed capital model, we provide in Section 2.2 a brief outline of the theory of circulating capital models.

\subsection{A single-product industries, circulating capital model}

The price equations, analogous to ( $3 \mathrm{~b})$ and (3d), for the single-product industries, circulating capital model of Part I of [8] are

$$
(1+r) p A+w l=p
$$

and

$$
p=w l[I-(1+r) A]^{-1}
$$

where $p$ is the vector of marketable commodity prices and $A$ is the matrix of input-output coefficients (see, for example, Chapter 5 of Pasinetti [6] or Chapter 3 of Woods [9]).

The fundamental condition to be imposed on a model is that it is productive. In the context of a single-product industries, circulating capital model, this condition is written as

$$
\exists x>0 \text { such that } A x<x
$$

where $x$ is interpreted as the vector of gross outputs, with $A x$ the vector of means of production used up in production. Assuming that all commodities are basic in the sense of Sraffa (i.e. that $A$ is indecomposable; see Newman [5], Chapter 5 of Pasinetti [6] or Chapter 3 of Woods [9]), (5) implies the the Frobenius root of $A$, $\lambda^{*}(A)$, is less than one. When $w=0,(4 \mathrm{a})$ becomes

$$
(1+R) q A=q,
$$

which has solution $R>0, q>\mathbf{0}$ by the Perron-Frobenius Theorem; $R=$ $1 / \lambda^{*}(A)-1>0$, and $[I-(1+r) A]^{-1}>O$ if and only if $1 /(1+r)>\lambda^{*}(A)$ $=1 /(1+R)$, i.e. if and only if $R>r$ (see Chapter 2 of Woods [9] or Chapter 5 of Passinetti [6]). So, (4b) has a positive solution $p$ if $r<R$, the maximum rate of profit, and $p=q$ when $r=R$. 
As the price vector is positive for all $r \leqslant R$, it follows that any commodity, say the $i$ th, can be chosen as numeraire. Post-multiplying by the column vector $e^{i}$, the $i$ th unit of vector of order $n,(4 \mathrm{~b})$ becomes

$$
p e^{i}=p_{i} \equiv 1=w_{(i)} l[I-(1+r) A]^{-1} e^{i}
$$

(where $w_{(i)}$ denotes the wage in terms of commodity $i$ ), whence

$$
w_{(i)}=1 / l[I-(1+r) A]^{-1} e^{i} .
$$

Equation (7b), the wage curve, is well defined, because the matrix inverse is positive if $0 \leqslant r<R$.

In (4)-(7), we have summarised very briefly some straightforward applications of the Perron-Frobenius Theorem to the single-product industries, circulating capital model. Given the question posed at the end of Section 2.1, it is reasonable to enquire if this Theorem is immediately applicable to the fixed capital model.

We have shown above that the productivity condition (5) underlies the analysis of prices in a circulating capital good model. By analogy, a productivity condition must first be specified for the fixed capital model. Such a condition can be written as

$$
\exists x>0 \text { such that } A_{N} x \leqslant B_{N} x,
$$

with strict inequality for marketable commodities.

In other words, there exists an intensity vector, $x$, such that the output of each marketable commodity is greater than the total inputs of that commodity into all processes. However, (8) by itself does not ensure the existence of a positive maximum rate of profit, $R_{N}$, as the solution of

$$
\left(1+R_{N}\right) q_{N} A_{N}=q_{N} B_{N},
$$

which is the analogue for the fixed capital model of (6) for the circulating capital model. Compare (9) and (6). In (9), $B_{N}$ is not diagonal. Compare (3d) and (4b). In $[I-(1+r) A]$, the diagonal elements are positive, the off-diagonal elements are nonpositive for all $r \leqslant R$; the theory of $M$-matrices applies (see Chapter 2 of Woods [9] for connections between the Perron-Frobenius Theorem and the theory of $M$-matrices). In $\left[B_{N}-(1+r) A_{N}\right.$ ], these sign conditions on the diagonal and the off-diagonal elements are not satisfied. In the circulating capital model, the productivity condition and the sign conditions ensure that the matrix inverse is positive for all $r<R$; in the fixed capital model, the sign conditions are not satisfied, so that the theory of $M$-matrices cannot be applied.

\subsection{The elimination of ageing machines}

In this section, we demonstrate that (3b) can be transformed into an equation to which the Perron-Frobenius Theorem (or, equivalently, the theory of $M$ matrices) can be applied. We follow the procedure suggested by Sraffa in Section 
76 of [8] to eliminate ageing machines. Consider (2), the processes for sector $(n+1)$. Multiply the $s$ th equation by $(1+r)^{N-s}, s=1, \ldots, N$, and add the resulting $N$ equations together to obtain

$$
\begin{gathered}
(1+r) p^{f} \sum_{s=1}^{N} A^{n+s}(1+r)^{N-s}+w_{N} \sum_{s=1}^{N} l_{n+s}(1+r)^{N-s} \\
=p_{n+1} \sum_{s=1}^{N} b_{n+s}(1+r)^{N-s} .
\end{gathered}
$$

Define,

$$
\begin{gathered}
\underline{A}^{n+1}=\sum_{s=1}^{N} A^{n+s}(1+r)^{N-s}, \quad \underline{l}_{n+1}=\sum_{s=1}^{N} l_{n+s}(1+r)^{N-s}, \\
\underline{b}_{n+1}=\sum_{s=1}^{N} b_{n+s}(1+r)^{N-s} .
\end{gathered}
$$

Combine (1) and (10), using (11), to obtain

$$
\begin{aligned}
(1+r) p^{f}\left[A^{1} \cdots\right. & \left.A^{n} \underline{A}^{n+1}\right]+w_{N}\left[l_{1} \cdots l_{n} \underline{l}_{n+1}\right] \\
& =p^{f}\left[e^{1} \cdots e^{n} \underline{b}_{n+1} e^{n+1}\right]
\end{aligned}
$$

where $e^{j}$ is the $j$ th unit vector of order $(n+1), j=1, \ldots, n+1$. Rewrite (12) as

$$
(1+r) p^{f} \underline{A}_{N}+w_{N} \underline{l}_{N}=p^{f} \underline{B}_{N},
$$

where

$$
\begin{aligned}
\underline{A}_{N} & =\left[\begin{array}{lll}
A^{1} & \cdots & A^{n} \underline{A}^{n+1}
\end{array}\right], \\
\underline{l}_{N} & =\left[\begin{array}{lll}
l_{1} & \cdots & l_{n} \underline{l}_{n+1}
\end{array}\right], \\
\underline{B}_{N} & =\left[\begin{array}{lll}
e^{1} & \cdots & e^{n} \underline{b}_{n+1} e^{n+1}
\end{array}\right] .
\end{aligned}
$$

Clearly, $\underline{B}_{N}$ is a positive diagonal matrix. Post-multiplying (13) by $\underline{B}_{N}^{-1}$ yields

$$
(1+r) p^{f} \underline{A}_{N} \underline{B}_{N}^{-1}+w_{n} \underline{l}_{N} \underline{B}_{N}^{-1}=p^{f},
$$

or

$$
(1+r) p^{f} \bar{A}_{N}+w_{N} \bar{l}_{N}=p^{f}
$$

where

$$
\overline{A_{N}}=\underline{A}_{N} \underline{B}_{N}^{-1}, \bar{l}_{N}=\underline{l}_{N} \underline{B}_{N}^{-1} .
$$

Equation (15) bears a superficial resemblance to (4a). Whereas $A$ and $l$ are constants in (4a), $\bar{A}_{N}$ and $\bar{l}_{N}$ are functions of $r$ in (15). So, further work remains to be done on (15) before the Perron-Frobenius Theorem can be applied.

Without loss of generality, $x$ can be replaced in (8) by 1 , the $(n+N)$ tuple of ones. Such a redefinition of units of measurement (so that each gross output is equal to one) can be achieved by a simple similarity transformation involving the 
positive diagonal matrix $X=\left[x_{i} \delta_{i j}\right]$. We can now rewrite the productivity condition embodied in (8) as:

Definition 1. Technique $(N)$, described by the triple $\left\{A_{N}, l_{n} ; B_{N}\right\}$ is productive if

$$
A_{N} \mathbf{1} \leqslant B_{N} \mathbf{1}
$$

with strict inequality for the first $(n+1)$ components.

In Section 2.2, it was demonstrated that the existence of a positive maximum rate of profit, $R$, could be derived for the circulating capital model on the basis of the productivity condition (5); it was assumed also that all commodities were basic or that the input-output matrix was indecomposable. We now demonstrate that a fixed capital model which is productive in the sense of Definition 1 also has a positive maximum rate of profit, $R_{N}$; as in the circulating capital case, we assume that the model is indecomposable.

THEOREM 1. In a productive single-product industries, fixed capital model, there exists a positive maximum rate of profit, $R_{N}$, such that the price subvector of marketable commodities is positive for all $r \in\left[0, R_{N}\right]$.

Proof. We derive this result in four stages.

(i) (15) refers only to marketable commodities. To apply Definition 1 to (15), it is necessary to eliminate ageing machines. To achieve this, apply the procedure suggested by Sraffa with $r=0$. Equation (17) is then converted into

$$
\underline{A}_{N}(0) 1<\underline{B}_{N}(0) 1,
$$

where $\underline{A}_{N}(0)$ denotes the fact that the elements of $\underline{A}_{N}$, which are functions of $r$, have been evaluated at $r=0$; similarly, for $\underline{B}_{N}(0)$. Equation (18) merely states that the economy is capable of producing a physical surplus of each marketable commodity over and above inter-industry requirements. As $\underline{B}_{N}(0)$ is a positive diagonal matrix, it follows from (18) that

$$
\underline{B}_{N}(0)^{-1} \underline{A}_{N}(0) \mathbf{1}<\mathbf{1} \text {. }
$$

As $\underline{A}_{N}(0) \geqslant O$, it follows from (19) that the Frobenius root of $\underline{B}_{N}(0)^{-1} \underline{A}_{N}(0)$, denoted by $\lambda^{*}(0)$, is less than one, using Theorem 18 in Chapter 2 of Woods [9].

(ii) The analysis of (15) is concerned with the matrix $\bar{A}_{N}=\underline{A}_{N} \underline{B}_{N}^{-1} \geqslant O$. Consider the roots of the matrix $(1+r) \overline{A_{N}}$. By the Perron-Frobenius Theorem, there exists corresponding to each $r>0$, the Frobenius root, $\phi(r)>0$, and the 
Frobenius vector, $q(r)>\mathbf{0}$, such that

$$
\phi(r) q(r)=q(r)(1+r) \overline{A_{N}}(r)
$$

where $\overline{A_{N}}(r)$ emphasises the functional dependence on $r$. When $r=0$, (20) becomes

$$
\begin{aligned}
\phi(0) q(0) & =q(0) \overrightarrow{A_{N}}(0) \\
& =q(0) \underline{A}_{N}(0) \underline{B}_{N}(0)^{-1} .
\end{aligned}
$$

Now,

$$
\underline{B}_{N}(0) \underline{B}_{N}(0)^{-1} \underline{A}_{N}(0) \underline{B}_{N}(0)^{-1}=\underline{A}_{N}(0) \underline{B}_{N}(0)^{-1},
$$

so that $\underline{A}_{N}(0) \underline{B}_{N}(0)^{-1}$ and $\underline{B}_{N}(0)^{-1} \underline{A}_{N}$ are similar. As similar matrices have the same characteristic roots, it follows that

$$
\phi(0)=\lambda^{*}(0)<1 .
$$

(iii) We now demonstrate that $\phi(r) \rightarrow \infty$ as $r \rightarrow \infty$. By indecomposability, at least one component of the column vector $A^{n+1}$ is positive; so $A^{n+1} \geqslant 0$. It then follows from (11) that $\underline{A}^{n+1}$ is a vector of polynomials in $(1+r)$, at least one component of which has degree $(N-1)$. Also from $(11), \underline{b}_{n+1}$ is a polynomial in $(1+r)$ of degree $(N-1)$, if $b_{n+1}>0 . \underline{A}^{n+1} / \underline{b}_{n+1}$ is thus a vector of rational polynomials in $(1+r)$. Let,

$$
\frac{\underline{A}^{n+1}}{\underline{b}_{n+1}}=\frac{F(1+r)}{g(1+r)}=\left[\frac{F_{i}(1+r)}{g(1+r)}\right], \quad i=1, \ldots, n+1 .
$$

From above,

$$
\operatorname{deg} F_{i}(1+r) \leqslant \operatorname{deg} g(1+r),
$$

with at least one equality, $i=1, \ldots, n+1$, and where $\operatorname{deg} g(1+r)$ is the degree of the polynomial $g(1+r)$.

Now consider $\overline{A_{N}}=\underline{A}_{N} \underline{B}_{N}^{-1}$. From (14), the first $n$ column of $\overline{A_{N}}$ are constant and so are unaffected by any change in $r$. The components of the $(n+1)$ th column are obviously affected by changes in $r$. There are two cases to examine:

(a) If $\operatorname{deg} F_{i}(1+r)<\operatorname{deg} g(1+r)$, it follows that $F_{i}(1+r) / g(1+r) \rightarrow 0$ as $r \rightarrow \infty$.

(b) If $\operatorname{deg} F_{i}(1+r)=\operatorname{deg} g(1+r)$, it follows that $F_{i}(1+r) / g(1+r) \rightarrow \bar{a}_{n+1, i}$ as $r \rightarrow \infty$, where $\bar{a}_{n+1, i}$ is a constant.

As (b) holds for at least one $i$, it follows that the $(n+1)$ th column of $\overline{A_{N}}$ tends towards a semipositive vector as $r \rightarrow \infty$. The first $n$ columns of $\bar{A}_{N}$ are constant as $r \rightarrow \infty$. By indecomposability, each row (or column) sum of $\bar{A}_{N}$ is positive for any $r \geqslant 0$. Hence, as $r \rightarrow \infty$, each row (or column) sum of $(1+r) \overline{A_{N}} \rightarrow \infty$. From Theorem 67 in Chapter 2 of Woods [9], the Frobenius root of a matrix lies between the largest and smallest row (or column) sums. As each row (or column) 
sum of $(1+r) \overline{A_{N}}$ tends to infinity with $r$, it follows that

$$
\phi(r) \rightarrow \infty \text { as } r \rightarrow \infty \text {. }
$$

(iv) We complete the proof by observing first of all that $\phi(r)$ is a continuous function of the elements of $(1+r) \overline{A_{N}}$. Using (23) and (26), it then follows that there exists at least one positive value of $r$ such that $\phi(r)=1$. Let the smallest such value of $r$ be denoted by $R_{N}$. Hence,

$$
\phi(r)<1 \quad \text { for } 0 \leqslant r<R_{N}, \quad \phi\left(R_{N}\right)=1 .
$$

The theorem refers only to marketable commodity prices; so consider (15). From (27), $\phi(r)<1$ for $0 \leqslant r<R_{N}$. Hence,

$$
\left[I-(1+r) \overline{A_{N}}\right]^{-1}>O \quad \text { if and only if } r<R_{N} \text {. }
$$

So,

$$
p^{f}=w_{N} \bar{l}_{N}\left[I-(1+r) \bar{A}_{N}\right]^{-1}>\mathbf{0} \quad \text { if } 0 \leqslant r<R_{N} .
$$

When $r=R_{N}, p^{f}=q^{f}\left(R_{N}\right)>0$, where

$$
q^{f}\left(R_{N}\right)=q^{f}\left(R_{N}\right)\left(1+R_{N}\right) \overline{A_{N}} .
$$

We defined $R_{N}$ in (9) as the maximum uniform rate of profit for the whole system, that is, including both marketable and nonmarketable commodities. The existence of such a value of the rate of profit cannot be assumed; it must be derived. Theorem 1 is the first part of such an existence proof. In this Theorem, we have derived the existence of a maximum uniform rate of profit, denoted by $R_{N}$, for a subsystem consisting only of marketable commodities. In Section 2.4, we demonstrate the significance of $R_{N}$ for all prices (given the indecomposability of the system, such a result is intuitively reasonable). $R_{N}$, defined in (27), turns out to be identical with that in (9).

\subsection{A non-singularity theorem}

In Section 2.3, it has been demonstrated that, by performing a series of elementary operations, the price equations (36) can be reduced to (15), which involve only marketable commodity prices. In Theorem 1 , we demonstrated the significance of $R_{N}$ for marketable commodity prices. $R_{N}$ is the smallest positive rate of profit for which the matrix $\left[I-(1+r) \overline{A_{N}}\right]$ is singular. Now,

$$
\begin{aligned}
I-(1+r) \bar{A}_{N} & =I-(1+r) \underline{A}_{N} \underline{B}_{N}^{-1} \\
& =\left[\underline{B}_{N}-(1+r) \underline{A}_{N}\right] \underline{B}_{N}^{-1} .
\end{aligned}
$$

As $\underline{B}_{N}^{-1}$ is a positive diagonal matrix for all $r \geqslant 0$, it follows from (31) that $\left[\underline{B}_{N}-(1+r) \underline{A}_{N}\right]^{-1}>O$ if and only if $\left[I-(1+r) \bar{A}_{N}\right]^{-1}>O$. This is merely a 
restatement of the fact that (13) and (15) have the same solutions. Rewrite (13) as

$$
p^{f}\left[\underline{B}_{N}-(1+r) \underline{A}_{n}\right]=w_{n} \underline{l}_{N}
$$

whence

$$
p^{f}=w_{N} \underline{l}_{N}\left[\underline{B}_{N}-(1+r) \underline{A}_{N}\right]^{-1}>\mathbf{0}
$$

if $0 \leqslant r<R_{N}$. Equation (13) or (32) relates only to marketable commodity prices. The analogous equation for the complete system is given by equation $(3 \mathrm{c})$,

$$
p_{N}\left[B_{N}-(1+r) A_{N}\right]=w_{N} l_{N} \text {. }
$$

We now demonstrate that $\left[B_{N}-(1+r) A_{N}\right]$ is nonsingular if $0 \leqslant r<R_{N}$. Then (3c) has a solution

$$
p_{N}=w_{N} l_{N}\left[B_{N}-(1+r) A_{N}\right]^{-1}
$$

over exactly the same range of values of $r$ as (32), or (13), has a solution.

For ease of notation, let

$$
C_{N}=B_{N}-(1+r) A_{N}
$$

Explicitly,

$$
C_{N}=\left[\begin{array}{ccccccccc}
C^{1} & \ldots & C^{n} & C^{n+1} & C^{n+2} & C^{n+3} & & \ldots & C^{n+N} \\
0 & \ldots & 0 & 1 & -(1+r) & 0 & & \ldots & 0 \\
0 & \ldots & 0 & 0 & 1 & -(1+r) & 0 & \ldots & 0 \\
. & & \cdot & \cdot & \cdot & & . & & . \\
\cdot & & \cdot & \cdot & & & & & 0 \\
0 & \ldots & 0 & 0 & 0 & & 0 & 1 & -(1+r)
\end{array}\right]
$$

where

$$
\begin{aligned}
C^{j} & =e^{j}-(1+r) A^{j}, \quad j=1, \ldots, n, \\
C^{n+v} & =b_{n+v} e^{n+1}-(1+r) A^{n+v}, \quad v=1, \ldots, N,
\end{aligned}
$$

( $e^{j}$ is the $j$ th unit vector of order $\left.(n+1), j=1, \ldots, n+1\right)$. Each of the column vectors $C^{j}$ has $(n+1)$ components. There are $(N-1)$ terms, $-(1+r)$, on the main diagonal, and $(N-1)$ terms, 1 , on the subdiagonal. We now prove:

Theorem 2. $C_{N}$ is nonsingular for all $r \in\left[0, R_{n}\right)$. In fact, sign $\operatorname{det} C_{N}=$ $\operatorname{sign}(-1)^{N-1}$.

Proof. We use the elementary fact that a matrix is nonsingular if and only if its determinant is nonzero. 


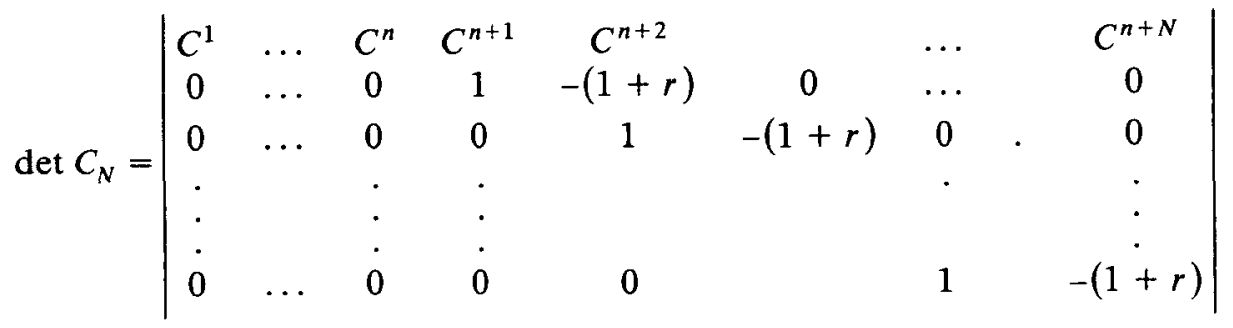

Multiply column $(n+v)$ by $(1+r)^{N-v}, v=1, \ldots, N$, to obtain

$$
\begin{aligned}
& \operatorname{det} C_{N}=\left[1 /(1+r)^{\sum_{v-1}^{N}(N-v)}\right] .
\end{aligned}
$$

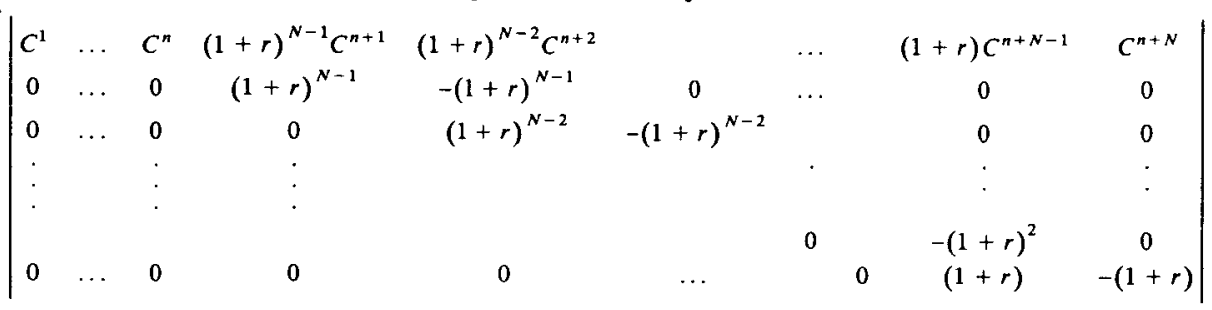

Add column $(n+N)$ to column $(n+N-1)$;

$$
\begin{aligned}
& \operatorname{det} C_{n}=\left[1 /(1+r)^{\sum_{v-1}^{N}(N-v)}\right] . \\
& \left|\begin{array}{cccccccccc}
C^{1} & \ldots & C^{n} & (1+r)^{N-1} C^{n+1} & (1+r)^{N-2} C^{n+2} & & \ldots & & \tilde{C}^{n+N-1} & C^{n+N} \\
0 & \cdots & 0 & (1+r)^{N-1} & -(1+r)^{N-1} & 0 & \ldots & & 0 & 0 \\
0 & \cdots & 0 & 0 & (1+r)^{N-2} & -(1+r)^{N-2} & 0 & \ldots & 0 & 0 \\
\vdots & & \vdots & \vdots & \vdots & & & & \vdots & \vdots \\
0 & \cdots & 0 & 0 & 0 & \ldots & 0 & \left(1+r^{2}\right) & -(1+r)^{2} & 0 \\
0 & \cdots & 0 & 0 & 0 & \ldots & & & 0 & -(1+r)
\end{array}\right|
\end{aligned}
$$

$$
\text { where } \tilde{C}^{n+N-1}=(1+r) C^{n+N-1}+C^{n+N} .
$$

The effect of this operation is to convert $(1+r) C^{n+N-1}$ into $\tilde{C}^{n+N-1}$ and to replace the $(1+r)$ in the $(n+N, n+N-1)$ position by 0 . If we now add column $(n+N-1)$ to column $(n+N-2)$ in (38), the effect will be to replace $(1+r)^{2} C^{n+N-2}$ by

$$
\tilde{C}^{n+N-2}=(1+r)^{2} C^{n+N-2}+\tilde{C}^{n+N-1}
$$

and the $(1+r)^{2}$ term in the $(n+N-1, n+N-2)$ position by 0 .

In general, the process can be described as follows. To column $(n+v)$, add columns $n+v+1, \ldots, n+N$. The effect of this is to convert $(1+r)^{N-v} C^{n+v}$ 
into $\tilde{C}^{n+v}$, where

$$
\tilde{C}^{n+v}=\sum_{s=0}^{N-v} C^{n+v+s}(1+r)^{N-v-s}
$$

and the term $(1+r)^{N-v}$ in the $(n+N+v-1, n+N-v)$ position to 0 . As these elementary operations do not affect the value of the determinant, we obtain

$$
\begin{array}{|ccccccccc}
\multicolumn{8}{c}{\operatorname{det} C_{N}=\left[1 /(1+r)^{\sum_{v-1}^{N}(N-v)}\right] .} \\
C^{1} & \ldots & C^{n} & \tilde{C}^{n+1} & \tilde{C}^{n+2} & & \ldots & \tilde{C}^{n+N-1} & C^{n+N} \\
0 & \ldots & 0 & 0 & -(1+r)^{N-1} & 0 & \ldots & 0 & 0 \\
\vdots & & \vdots & \vdots & \vdots & & & \vdots & \vdots \\
0 & \ldots & 0 & 0 & 0 & \ldots & 0 & -(1+r)^{2} & 0 \\
0 & \ldots & 0 & 0 & 0 & \ldots & & 0 & -(1+r)
\end{array} \mid
$$

where $\tilde{C}^{n+o}$ is defined in (41).

There is now only one nonzero entry in each of rows $n+2, \ldots, n+N$. Factor $(1+r)$ from row $(n+N),(1+r)^{2}$ from row $(n+N-1)$ and so on until $(1+r)^{N-1}$ is factored from row $(n+2)$. Equation (42) then becomes

$$
\begin{aligned}
& \operatorname{det} C_{N}=\left[1 /(1+r)^{\sum_{v-1}^{N}(N-v)}\right] \cdot\left[(1+r)^{\sum_{v=1}^{N-1}(N-v)}\right] . \\
& \left|\begin{array}{cccccccc}
C^{1} & \cdots & C^{n} & \tilde{C}^{n+1} & \tilde{C}^{n+2} & \ldots & C^{n+N} \\
0 & \cdots & 0 & 0 & -1 & 0 & 0 \\
\vdots & & \vdots & \vdots & \vdots & & \vdots \\
0 & \ldots & 0 & 0 & 0 & 0 & 0 & -1
\end{array}\right| .
\end{aligned}
$$

Note that

$$
\sum_{v=1}^{N}(N-v)=\sum_{v=1}^{N-1}(N-v)+(N-N)=\sum_{v=1}^{N-1}(N-v)
$$

So,

$$
\operatorname{det} C_{N}=\left|\begin{array}{cccccccc}
C^{1} & \ldots & C^{n} & \tilde{C}^{n+1} & \tilde{C}^{n+2} & & \ldots & C^{n+N} \\
0 & \ldots & 0 & 0 & -1 & 0 & & 0 \\
\vdots & & \vdots & \vdots & \vdots & & & \vdots \\
0 & \ldots & 0 & 0 & 0 & \ldots & 0 & -1
\end{array}\right|
$$

Expanding in cofactors along row $(n+N)$, we obtain

$$
\operatorname{det} C_{N}=(-1)\left|\begin{array}{cccccccc}
C^{1} & \ldots & C^{n} & \tilde{C}^{n+1} & \tilde{C}^{n+2} & \ldots & \ldots & \tilde{C}^{n+N-1} \\
0 & \ldots & 0 & 0 & -1 & 0 & \ldots & 0 \\
\vdots & & \vdots & \vdots & \vdots & & & \vdots \\
0 & \ldots & 0 & 0 & 0 & \ldots & 0 & -1
\end{array}\right|
$$


Expanding in cofactors along row $(n+N-1)$, then row $(n+N-2)$ and so on until row $(n+2)$, we obtain

$$
\operatorname{det} C_{N}=(-1)^{N-1} \cdot \operatorname{det}\left[C^{1} \ldots C^{n} \tilde{C}^{n+1}\right] .
$$

From (36), $C^{j}=e^{j}-(1+r) A^{j}, j=1, \ldots, n$. From (40),

$$
\begin{aligned}
\tilde{C}^{n+1} & =\sum_{s=0}^{N-1} C^{n+1+s}(1+r)^{N-1-s} \\
& =\sum_{s=1}^{N} C^{n+s}(1+r)^{N-s} \\
& =\sum_{s=1}^{N}\left[b_{n+s} e^{n+1}-(1+r) A^{n+s}\right](1+r)^{N-s} \\
& =\left\{\sum_{s=1}^{N} b_{n+s}(1+r)^{N-s}\right\} e^{n+1}-(1+r) \sum_{s=1}^{N} A^{n+s}(1+r)^{N-s} \\
& =\underline{b}_{n+1} e^{n+1}-(1+r) \underline{A}^{n+1},
\end{aligned}
$$

using (11). Hence, from (14), (36) and (47),

$$
\begin{aligned}
{\left[C^{1} \ldots C^{n} \tilde{C}^{n+1}\right] } & =\underline{B}_{N}-(1+r) \underline{A}_{N} \\
& =\left[I-(1+r) \underline{A}_{N} \underline{B}_{N}^{-1}\right] \underline{B}_{N}=\left[I-(1+r) \bar{A}_{N}\right] \underline{B}_{N} .
\end{aligned}
$$

Using (48) in (46),

$$
\operatorname{det} C_{N}=(-1)^{N-1} \cdot \operatorname{det}\left[I-(1+r) \overline{A_{N}}\right] \cdot \operatorname{det} \underline{B}_{N},
$$

where $\operatorname{det} \underline{B}_{N}>0$ for all $r \geqslant 0$. From Theorem $1, \operatorname{det}\left[I-(1+r) \overline{A_{N}}\right]>0$ if $r \in$ $\left[0, R_{N}\right)$ and $\operatorname{det}\left[I-(1+r) \bar{A}_{N}\right]=0$ if $r=R_{N}$. The result now follows from (49).

As remarked in Section 2.2, $C_{N}$ is not an $M$-matrix because it does not satisfy the appropriate sign conditions. Consequently, though it exists for all $r \in\left[0, R_{N}\right)$, $C_{N}^{-1}$ may contain negative entries. This raises the possibility that $p_{N}$ contains negative entries; as marketable commodity prices are positive for all $r \in\left[0, R_{N}\right)$, it follows that negative prices must refer to ageing machines. To fix ideas, it is worthwhile at this stage to consider the phenomenon of negative ageing machine prices in the context of a simple example.

\subsection{A special case}

Consider the following two-sector model: a new machine is produced by means of inputs of iron (circulating capital) and labour; iron is produced by means of a machine (fixed capital), which lasts for two periods, iron and labour. With reference to the model described in Section 2.1, in particular (36), $n+1=2$ and 
$N=2$. We index commodities in the following way:

1 new machine

2 iron

3 one-year old mchine in the iron sector.

Employing the notation described in Section 2.1, we have as the price equations for this two-sector model,

$$
\begin{aligned}
& (1+r) p_{2} a_{21}+w l_{1}=p_{1}, \\
& (1+r) p_{2} a_{22}+(1+r) p_{1}+w l_{2}=p_{2} b_{2}+p_{3}, \\
& (1+r) p_{2} a_{23}+(1+r) p_{3}+w l_{3}=p_{2} b_{3} .
\end{aligned}
$$

Every commodity in this model is basic in the sense of Sraffa. As long as the model described in (50) satisfies a productivity condition, it follows, by applying the procedure described in Section 2.3, that there exits a positive maximum rate of profit, denoted by $R_{2}$.

Now we must consider the possibility that it may not be optimal to activate both of the processes available to the iron sector in (50). In other words, the iron sector may scrap the machine after the first process of production; the iron industry operates only its first process. In such a case, the price equations are given by:

$$
\begin{aligned}
& (1+r) p_{2} a_{21}+w l_{1}=p_{1}, \\
& (1+r) p_{2} a_{22}+(1+r) p_{1}+w l_{2}=p_{2} b_{2} .
\end{aligned}
$$

(It is assumed that the machine can be scrapped costlessly; it should be pointed out that the incorporation of scrap values does not present any new conceptual, as opposed to notational, problems). In effect, (51), describes a circulating capital model; to obtain a system analogous to (4a), we merely divide the second equation of (51) by $b_{2}$. If the model in (51) satisfies a productivity condition, a positive maximum rate of profit, denoted by $R_{1}$, exists, by the theory outlined in Section 2.2.

The point to be realised is that even in this simple model, summarised in (50) and (51), the question of choice of technique arises. In this Section, we provide a preliminary discussion of this question in the context of the simple two-sector model; denote the technique in (50) by (2), that in (51) by (1). Consider (2) first. In Section 2.3, we demonstrated the procedure for eliminating ageing machines. From (50), we obtain

$$
(1+r) p^{f} \underline{A}_{2}+w_{2} \underline{l}_{2}=p^{f} \underline{B}_{2},
$$

or

$$
(1+r) p^{f} \bar{A}_{2}+w_{2} \bar{l}_{2}=p^{f},
$$


or

$$
p^{f}=w_{2} i_{2}\left[I-(1+r) \overline{A_{2}}\right]^{-1}>0 \text { if } 0 \leqslant r<R_{2} .
$$

With the $i$ th commodity as numeraire, $i=1$ or 2 , we obtain from (53)

$$
p^{f} e^{i}=p_{i} \equiv 1=w_{2}^{(i)} \tilde{l}_{2}\left[I-(1+r) \bar{A}_{2}\right]^{-1} e^{i}
$$

or

$$
w_{2}^{(i)}=1 / i_{2}\left[I-(1+r) \bar{A}_{2}\right]^{-1} e^{i},
$$

where $w_{2}^{(i)}$ denotes the wage of technique (2) with respect to the $i$ th commodity as numeraire ( $e^{i}$ is the $i$ th vector of order 2).

Now consider technique (1). After dividing the second equation of (51) by $b_{2}$, we obtain,

$$
(1+r) p^{f} \overline{A_{1}}+w_{1} \bar{l}_{1}=p^{f}
$$

or

$$
p^{f}=w_{1} \bar{l}_{1}\left[I-(1+r) \overline{A_{1}}\right]^{-1}>0 \quad \text { if } 0 \leqslant r<R_{1} .
$$

With the $i$ th commodity as numeraire, $i=1$ or 2 , we obtain from (56)

$$
p^{f} e^{i}=p_{i} \equiv 1=w_{1}^{(i)} \bar{l}_{1}\left[I-(1+r) \overline{A_{1}}\right]^{-1} e^{i}
$$

or

$$
w_{1}^{(i)}=1 / \ddot{l}_{1}\left[I-(1+r) \bar{A}_{1}\right]^{-1} e^{i}
$$

If we choose the same numeraire in (54) and (57), we can draw the wage curves of both techniques on the same diagram. Consider Figure 1, which is based on this set of data:

$$
\begin{array}{lll}
a_{21}=1, & l_{1}=1, & \\
a_{22}=2, & l_{2}=2, & b_{2}=15 \\
a_{23}=3, & l_{3}=s 3, & b_{3}=12 .
\end{array}
$$

$R_{1}=2, R_{2}=2.15$; with the second commodity as numeraire, the maximum wage rates in the two techniques are $W_{1}^{(2)}=4, W_{2}^{(2)}=7 / 2$. It can be seen from Figure 1 that there is one point of intersection between $w_{1}^{(2)}$ and $w_{2}^{(2)}$ in the positive quadrant. This point of intersection, or switch-point, occurs at $r^{*}=0.75$. (1) is chosen for $r \in\left[0, r^{*}\right)$ and (2) for $r \in\left(r^{*}, R_{2}\right]$, at $r^{*}$, either (1) or (2) or a convex combination of both can be chosen (see Chapter 6 of Pasinetti [6] or Chapter 3 of Woods [9] for a summary of results on choice of technique; it is shown there that a particular technique is chosen at a given value of $r$ if it supports the highest real wage rate). 


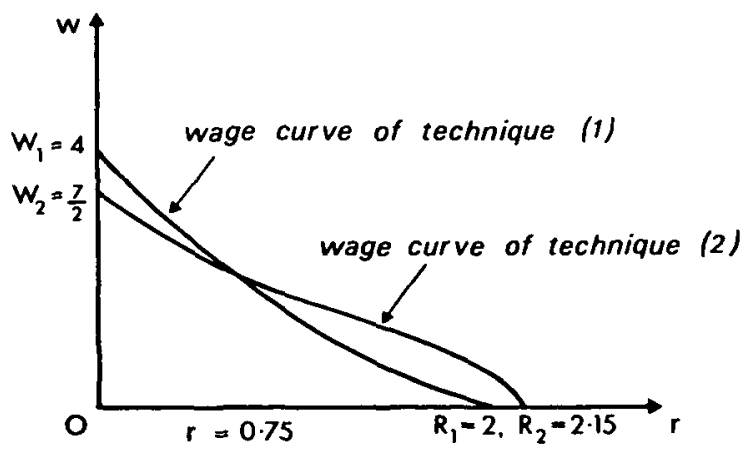

Figure 1. An example of switching of techniques in a fixed capital model.

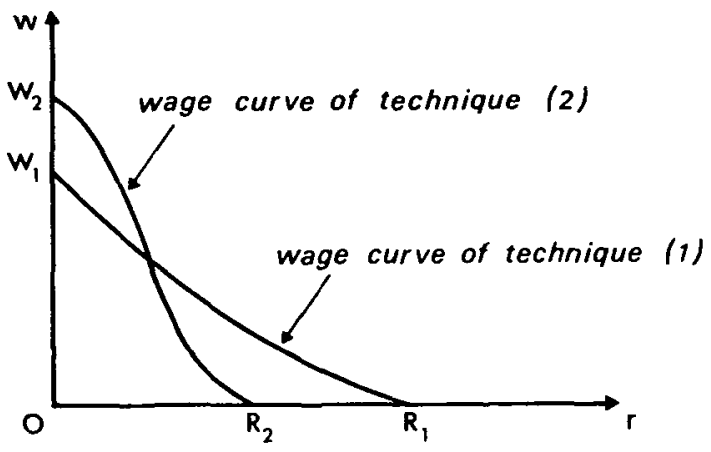

Figure 2. A second example of switching of techniques.

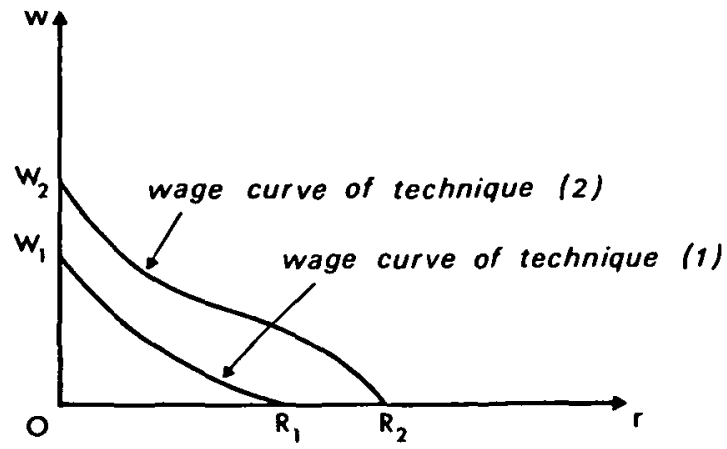

Figure 3. Technique (2) dominates technique (1). 


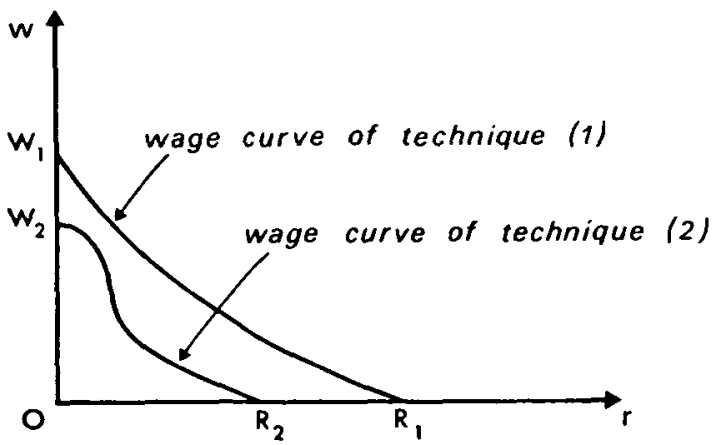

Figure 4. Technique (1) dominates technique (2).

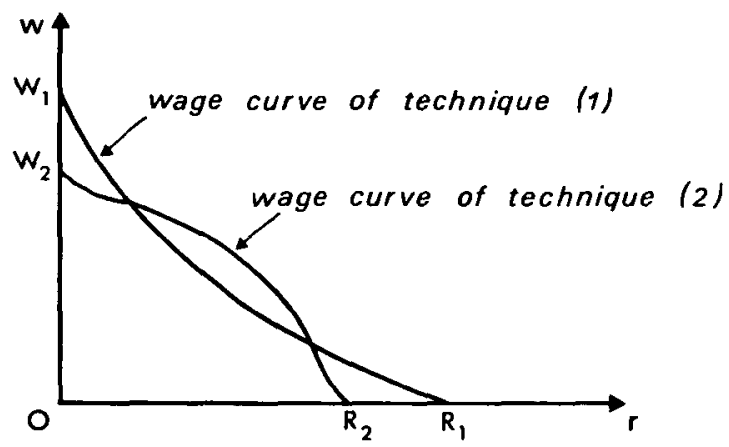

Figure 5. An example of reswitching of techniques in a fixed capital model.

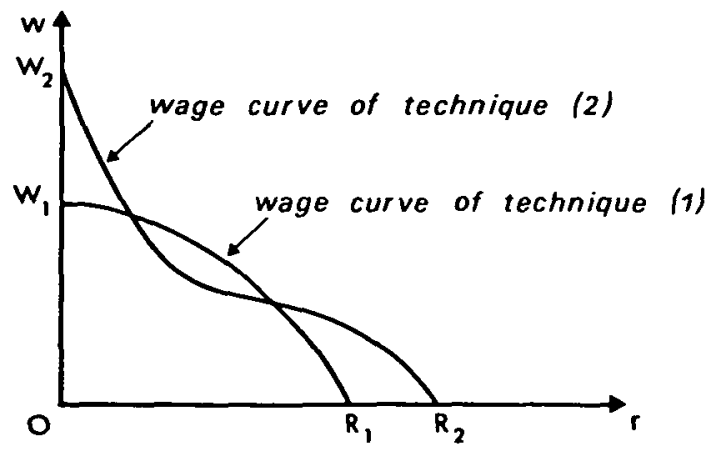

Figure 6. A second example of reswitching of techniques. 
There are three remarks to be made here:

REMARK 3. For that sub-interval of $\left[0, R_{2}\right]$ over which (1) is chosen, the price of the ageing machine, $p_{3}$, is negative.

REMARK 4. There is not a inverse monotonic relation between the optimal economic lifetime of a machine and the rate of profit. This is clearly demonstrated in Figure 1, where the optimal economic lifetime of the machine is one period for $r \in\left[0, r^{*}\right)$ and two periods for $r \in\left(r^{*}, R_{2}\right]$.

RemarK 5. Any ordering of techniques is possible, as illustrated in Figures 1-6. It is possible to construct numerical examples to illustrate each of the patterns in Figures 2 to 6.

We shall discuss Remark 3 at length in Sections 2.6 and 2.8 in the context of the model outlined in Section 2.1.

\subsection{Three theorems on fixed capital matrices}

After the digression in Section 2.5 to consider a special case, we now take up the discussion initiated in Sections 2.3 and 2.4. The aim in this section is to derive further preliminary results, prior to the analysis of negative ageing machine prices in Section 2.8.

Consider the price vector given by equation ( $3 \mathrm{~d})$;

$$
p_{N}=w_{N} l_{N}\left[B_{N}-(1+r) A_{N}\right]^{-1}=w_{N} l_{N} C_{N}^{-1}
$$

where the inverse exists for all $r \in\left[0, R_{N}\right)$ by Theorem 2. From Theorem 1 , the price subvector of marketable commodity prices is positive over this interval. So, postmultiplying (3d) by $e^{i}$, the $i$ th unit vector of order $(n+N), i=1, \ldots, n+1$, we obtain,

$$
p_{N} e^{i}=p_{i} \equiv 1=w_{N}^{(i)} l_{N} C_{N}^{-1} e^{i}
$$

where the $i$ th commodity is chosen as numeraire. By analogy with (7b) in Section 2.2 , it is reasonable to expect that

$$
w_{N}^{(i)}=1 / l_{N} C_{N}^{-1} e^{i}
$$

is the wage curve of technique $(N)$. The possibility of negative entries in the matrix $C_{N}^{-1}$ has already been referred to. So, to establish that the operation described in (61) can be performed, we prove:

THEOREM 3. The first $(n+1)$ columns of $C_{N}^{-1}$ are positive. 
Proof. We have already obtained an expression for $\operatorname{det} C_{N}$ in Theorem 2. To prove this Theorem, we examine the first $(n+1)$ columns of adj $C_{N}$, the adjoint matrix of $C_{N}$.

Let $C_{i j}$ denote the cofactor of the $(i, j)$ element of $C_{N}, i=1, \ldots, n+1$, $j=1, \ldots, n+N$.

First of all, suppose that $j<n+1$.

$C_{i j}=(-1)^{i+j}$

\begin{tabular}{|ll|llllll|}
$c_{11}$ & $\cdots$ & $\cdot$ & $c_{1, n+1}$ & $c_{1, n+2}$ & $\cdots$ & & $c_{1, n+N}$ \\
$\vdots$ & & & $\vdots$ & $\vdots$ & & & $\vdots$ \\
\hline$\vdots$ & & & $\vdots$ & $\vdots$ & & & $\vdots$ \\
$c_{n+1,1}$ & $\cdots$ & $\cdot$ & $c_{n+1, n+1}$ & $c_{n+1, n+2}$ & $\cdots$ & & $c_{n+1, n+N}$ \\
0 & $\cdots$ & $\cdot$ & 1 & $-(1+r)$ & 0 & $\ldots$ & 0 \\
$\vdots$ & & $\vdots$ & $\vdots$ & & & $\vdots$ \\
0 & $\ldots$ &. & 0 & 0 & $\cdots$ & & $1-(1+r)$
\end{tabular}

(the lines indicate the row and column to be deleted). By repeating the procedure used in Section 2.4 to prove Theorem 2, we obtain

$C_{i j}=(-1)^{i+j}$

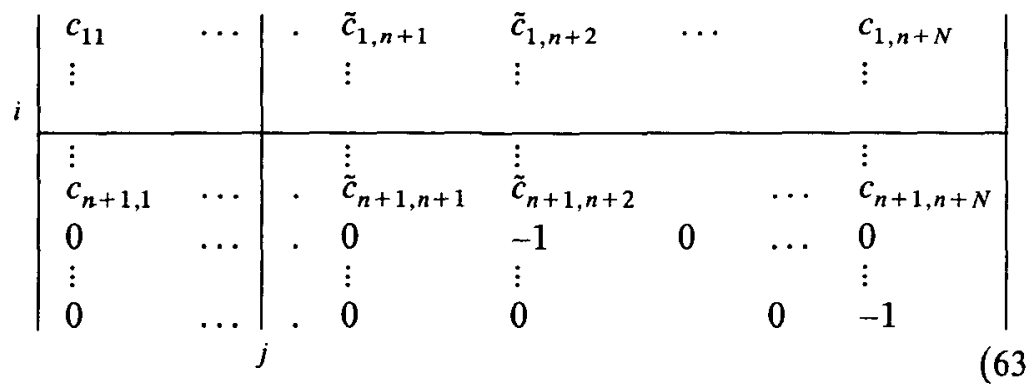

where

$$
\tilde{C}^{n+v}=\left[\begin{array}{c}
\tilde{c}_{1, n+v} \\
\tilde{c}_{2, n+v} \\
\vdots \\
\tilde{c}_{n+1, n+v}
\end{array}\right] .
$$


Expanding (63) in cofactors along row $(n+N-1)$, then along row $(n+N-2)$ and so on until row $(n+1)$, we obtain

$$
\begin{aligned}
C_{i j}=(-1)^{i+j}(-1)^{N-1} & \\
& \left|\begin{array}{ll|ll}
c_{11} & \ldots & . . & \tilde{c}_{1, n+1} \\
\vdots & & & \vdots \\
& \vdots & & \\
\hline c_{n+1, n+1} & \ldots & . . & \vdots \\
\tilde{c}_{n+1, n+1}
\end{array}\right|
\end{aligned}
$$

Now $(-1)^{i+j}$ times the determinant on the right-hand side of $(65)$ is the cofactor of the $(i, j)$ element of the matrix $\left[\underline{B}_{N}-(1+r) \underline{A}_{N}\right]$. This is an $M$-matrix because it satisfies the sign pattern and has a positive inverse (see Chapter 2 of Woods [9] for a summary of results on $M$-matrices). As $\operatorname{det}\left[\underline{B}_{N}-(1+r) \underline{A}_{N}\right]>0$, it follows that each cofactor of $\left[\underline{B}_{N}-(1+r) \underline{A}_{N}\right]$ is positive for all $r \in\left[0, R_{N}\right)$. Using this in (65), we conclude that

$$
\operatorname{sign} C_{i j}=\operatorname{sign}(-1)^{N-1} .
$$

From Theorem 2,

$$
\text { sign det } C_{N}=\operatorname{sign}(-1)^{N-1} \text {. }
$$

Hence, each of the first $n$ elements in each of the first $(n+1)$ columns of $C_{N}^{-1}$ is positive.

Now suppose that $j=n+1$.

$$
C_{i, n+1}=(-1)^{i+n+1}
$$

\begin{tabular}{|lllllll}
$c_{11}$ & $\ldots$ & $c_{1, n+1}$ & $c_{1, n+2}$ & $\ldots$ & & $c_{1, n+N}$ \\
$\vdots$ & & & $\vdots$ & & \\
\hline$\vdots$ & & & $\vdots$ & & & $\vdots$ \\
$c_{n+1,1}$ & $\ldots$ & $c_{n+1, n+1}$ & $c_{n+1, n+2}$ & $\ldots$ & & $c_{n+1, n+N}$ \\
0 & $\ldots$ & 1 & $-(1+r)$ & 0 & $\ldots$ & 0 \\
$\vdots$ & & $\mid$ & & & & $\vdots$ \\
0 & $\ldots$ & 0 & 0 & & 1 & $-(1+r)$
\end{tabular} \mid

To evaluate this determinant, apply once again the procedure developed in the proof of Theorem 2. Note that this application must differ from earlier ones because the $(n+1)$ th column is deleted from the matrix in (68). So, multiply 
column $(n+v)$ by $(1+r)^{N-v}, v=2, \ldots, N$. Add column $(n+N)$ to column $(n+N-1)$; add the resulting column to column $(n+N-2)$, and so on until all these columns have been added to column $(n+2)$. We then obtain

$C_{i, n+1}=(-1)^{i+n+1} \cdot\left[1 /(1+r)^{\sum_{v-1}^{N}(N-v-1)}\right]$.

\begin{tabular}{|c|c|c|c|c|c|c|}
\hline $\begin{array}{l}c_{11} \\
\vdots\end{array}$ & $\ldots$ & $c_{1, n+1}$ & $\begin{array}{l}\bar{c}_{1, n+2} \\
\vdots\end{array}$ & & & $\begin{array}{l}c_{1, n+N} \\
\vdots\end{array}$ \\
\hline $\begin{array}{l}\vdots \\
c_{n+1,1}\end{array}$ & $\ldots$ & $c_{n+1, n+1}$ & $\vdots_{\tilde{c}_{n+1, n+2}}$ & $\ldots$ & & $\bar{c}_{n+1, n+N}$ \\
\hline 0 & $\ldots 0$ & 1 & $-(1+r)^{N-1}$ & 0 & & \\
\hline 0 & $\ldots$ & $i$ & 1 & $-(1+r)^{N-2}$ & $\begin{array}{ll}0 & \ldots\end{array}$ & 0 \\
\hline 0 & $\ldots$ & $\begin{array}{l}1 \\
0\end{array}$ & $\begin{array}{l}\vdots \\
0\end{array}$ & $\begin{array}{l}\vdots \\
0\end{array}$ & 0 & $\begin{array}{l}\vdots \\
-(1+r)\end{array}$ \\
\hline
\end{tabular}

Factor $(1+r)$ from row $(n+N),(1+r)^{2}$ from row $(n+N-1)$ and so on until $(1+r)^{N-1}$ is factored from row $(n+2)$ to obtain

$$
\begin{aligned}
& C_{i, n+1}=(-1)^{i+n+1} \cdot\left[(1+r)^{\sum_{v=1}^{N-1}(N-v)} /(1+r)^{\sum_{v-1}^{N-2}(N-v-1)}\right] \text {. }
\end{aligned}
$$

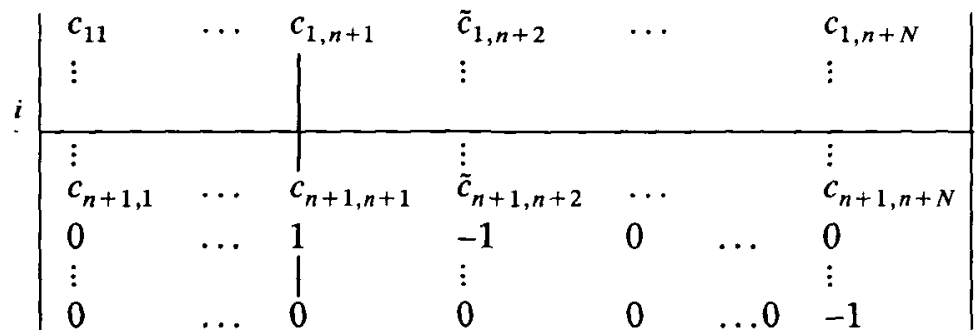

$$
\begin{aligned}
& =(-1)^{i+n+1} \cdot(-1)^{N-1} \cdot(1+r)^{N-1} \text {. } \\
& i\left|\begin{array}{lll}
c_{11} & \ldots & c_{1, n+1} \\
\vdots & & \\
\vdots & & \\
c_{n+1,1} & \ldots & c_{n+1, n+1}
\end{array}\right|
\end{aligned}
$$

as in (44)-(46). Now $(-1)^{i+n+1}$ times the determinant in (71) is the cofactor of the element $(i, n+1)$ of the matrix $\left[\underline{B}_{N}-(1+r) \underline{A}_{N}\right]$. By the same argument as above, this cofactor is positive for all $r \in\left[0, R_{N}\right)$. Hence,

$$
\operatorname{sign} C_{i, n+1}=\operatorname{sign}(-1)^{N-1} \text {. }
$$

From (66), (67) and (72), it follows that the $(n+1)$ th element in each of the first $(n+1)$ columns of $C_{N}^{-1}$ is positive. 
So far, we have proved that each of the first $(n+1)$ elements in each of the first $(n+1)$ columns of $C_{N}^{-1}$ is positive. We must now examine $C_{i, n+2}, \ldots, C_{i, n+N}$, $i=1, \ldots, n+1$. Consider the product

$$
C_{N} \cdot \operatorname{adj} C_{N}=\operatorname{det} C_{N} \cdot I_{n+N}
$$

There are only two nonzero entries, 1 and $-(1+r)$, in each of rows $n+2, \ldots, n$ $+N$. So, after multiplying each of rows $n+2, \ldots, n+N$ of $C_{N}$ by column $i$ of $\operatorname{adj} C_{N}$, we obtain

$$
\left.\begin{array}{l}
C_{i, n+1}-(1+r) C_{i, n+2}=0, \\
C_{i, n+2}-(1+r) C_{i, n+3}=0, \\
\vdots \\
C_{i, n+N-1}-(1+r) C_{i, n+N}=0,
\end{array}\right\}
$$

because $i$ can assume the values $1, \ldots, n+1$. Hence

$$
\begin{aligned}
\operatorname{sign} C_{i, n+1} & =\operatorname{sign} C_{i, n+2}=\cdots=\operatorname{sign} C_{i, n+N} \\
& =(-1)^{N-1}, \text { from (72). }
\end{aligned}
$$

Hence the last $(N-1)$ elements in the $i$ th column of $C_{N}^{-1}$ are all positive, $i=1, \ldots, n+1$. This completes the proof.

As suggested at the beginning of this section, Theorem 3 enables us to prove:

THEOREM 4. The wage curve of technique $(N)$ is given by (61), for any $i=$ $1, \ldots, n+1$.

Proof. Given the discussion prior to Theorem 3, it remains only to observe that, from Theorem 3, the denominator of (61) is well defined for all $r \in\left[0, R_{N}\right)$, for any choice of numeraire.

The final preliminary result on the fixed capital model is concerned with the existence of a Standard Commodity. This composite commodity has the property that it "consists of the same commodities (combined in the same proportions) as does the aggregate of its own means of production-in other words, such that both product and means of production are quantities of the self-same composite commodity." (Sraffa [8], Section 24, page 19) Sraffa's definition applies equally to a circulating as to a fixed capital model (for the derivation of the Standard Commodity in a single-product industries model, see Pasinetti [6], Chapter 5, or Woods [9], Chapter 3). 
Proof. $\underline{B}_{N}^{-1} \underline{A}_{N}$ is a semipositive indecomposable matrix that is similar to $\overline{A_{N}}=\underline{A}_{N} \underline{B}_{N}^{-1}$. So $\underline{B}_{N}^{-1} \underline{A}_{N}$ has Frobenius root $\left(1+R_{N}\right)^{-1}$ and corresponding positive characteristic vector $\underline{x}_{N}$ where

$$
\underline{x}_{N}=\left(1+R_{N}\right) \underline{B}_{N}^{-1} \underline{A}_{N} \underline{x}_{N}
$$

or

$$
\left[\underline{B}_{N}-\left(1+R_{N}\right) \underline{A}_{N}\right] \underline{x}_{N}=\mathbf{0},
$$

where $\underline{x}_{N}=\left[\underline{x}_{i}\right], i=1, \ldots, n+1$. Define

$$
x_{N}=\left[\underline{x}_{1}, \ldots, \underline{x}_{n},\left(1+R_{N}\right)^{N-1} \underline{x}_{n+1},\left(1+R_{N}\right)^{N-2} \underline{x}_{n+1}, \ldots,\left(1+R_{N}\right)^{0} \underline{x}_{n+1}\right]^{\prime}
$$

(where ' denotes transposition). Given (77) and (78), it follows by straightforward manipulation that

$$
\left[B_{N}-\left(1+R_{N}\right) A_{N}\right] x_{N}=\mathbf{0} .
$$

Equality in the first $(n+1)$ components of (79) follows from (77). Equality in the last $(N-1)$ components follows from the definition of $x_{N}$ in (5) and the fact that the matrix $\left[B_{N}-\left(1+R_{N}\right) A_{N}\right]$ has only two nonzero entries, 1 and $-(1+r)$, in each of its last $(N-1)$ rows.

The Standard Commodity, based on the vector $x_{N}$, is proportional to the vectors $B_{N} x_{N}$ and $A_{N} x_{N}$. It clearly satisfies Sraffa's definition that the output vector $\left(B_{N} x_{N}\right)$ is proportional to the vector of means of production $\left(A_{N} x_{N}\right)$.

Theorems 3-5 have been derived for technique $(N)$ under the productivity condition (17). In Section 2.5, we considered the special case where $n+1=2$ and $N=2$, demonstrating that it may not be optimal to operate the machine until the end of its physical liftime. $A$ fortiori, in technique $(N)$, it may not be optimal to operate the ageing machine in sector $(n+1)$ for $N$ periods. The question of choice of technique which arises for the simpler two-sector model of Section 2.5 must also arise for the more general $(n+1)$-sector model. However, before considering this question, we must first of all ascertain the number of alternative techniques and which of the results derived above are applicable to them. This is done in Section 2.7.

\subsection{Alternative techniques in the fixed capital model}

It was demonstrated in Section 2.5 that alternative techniques of production exist in a two-sector model where one sector employs an ageing machine with a maximum physical lifetime of two periods. In this simple model, there are two techniques of production, assuming that the other sector has only one production process. In the general $(n+1)$-sector model, assume that each of sectors $1, \ldots, n$ has only one process of production and that the machine used in sector $(n+1)$ has a maximum physical lifetime of $N$ periods. Then, there are $N$ techniques of 
production; the typical technique, denoted by $(h)$, is that in which the machine is employed in sector $(n+1)$ in the first $h$ processes only, $1 \leqslant h \leqslant N$. We use the word "deleted" to refer to techniques $(1), \ldots,(N-1)$. The price equations for technique $(h)$ are given by

$$
(1+r)\left[p^{f} A^{j}+p^{m} \mathbf{0}\right]+w l_{j}=p_{j}, \quad j=1, \ldots, n,
$$

and

$$
\left.\begin{array}{l}
(1+r)\left[p^{f} A^{n+1}+p^{m} 0\right]+w l_{n+1}=p_{n+1} b_{n+1}+p^{m} M^{1}, \\
(1+r)\left[p^{f} A^{n+2}+p^{m} M^{1}\right]+w l_{n+2}=p_{n+1} b_{n+2}+p^{m} M^{2} \\
\vdots \\
(1+r)\left[p^{f} A^{n+h}+p^{m} M^{h-1}\right]+w l_{n+h}=p_{n+1} b_{n+h}+p^{m} \mathbf{0} .
\end{array}\right\}
$$

It is assumed that ageing machines can be scrapped costlessly after $h$ production periods; hence, the presence of the $\mathbf{0}$ vector on the right-hand side of the last equaion in (80). Define

$$
\begin{aligned}
& A_{h}=\left[\begin{array}{ccc|ccc}
A^{1} & \ldots & A^{n} & A^{n+1} & \ldots & A^{n+h} \\
\mathbf{0} & \ldots & \mathbf{0} & M^{1} & \ldots & M^{h-1}
\end{array}\right], \\
& l_{h}=\left[l_{1} \ldots l_{n} \mid l_{n+1} l_{n+2} \ldots l_{n+h}\right] \text {, } \\
& B_{h}=\left[\begin{array}{ccc|cccc}
e^{1} & \ldots & e^{n} & \underline{e}^{n+1} & \ldots & \underline{e}^{n+h-1} & \underline{e}^{n+h} \\
\mathbf{0} & \ldots & \mathbf{0} & M^{1} & \ldots & M^{h-1} & 0
\end{array}\right],
\end{aligned}
$$

where $M^{v}$ is a unit vector of order $(h-1)$, as there are only $(h-1)$ ageing machines in this technique. Let

$$
p_{h}=\left(p_{1}, \ldots, p_{n}, p_{n+1} ; p_{n+2}, \ldots, p_{n+h}\right)=\left(p^{f} p^{m}\right) .
$$

Then (79) and (80) can be rewritten as

$$
(1+r) p_{h} A_{h}+w_{h} l_{h}=p_{h} B_{h} .
$$

Thus technique $(h)$ is defined by the triple $\left\{A_{h}, l_{h} ; B_{h}\right\} . A_{h}$ and $B_{h}$ are both square of order $(n+h) ; l_{h}$ and $p_{h}$ have $(n+h)$ components; $p^{f}$ has $(n+1)$ and $p^{m}$ has $(h-1)$ components.

The analysis in Section 2.4 is based on the assumption that technique $(N)$ is productive, as in Definition 1 . The analogous productivity condition for technique $(h)$ is

$$
A_{h} 1 \leqslant B_{h} 1 \text {, }
$$

with strict inequality for each of the first $(n+1)$ components.

Theorems 1-5 apply, mutatis mutandis, to any technique $(h)$ which satisfies productivity conditions such as (84). In Section 2.8 , it is assumed for ease of notation that each technique $(h)$ is productive, $h=1, \ldots, N$. This means that technique $(h)$ can support a positive maximum uniform rate of profit, $R_{h}$, such 
that $w_{h}>0$ if $r<R_{h}$ and $p^{f}>0$ if $r \leqslant R_{h}$. No assumptions are made about the ordering of either maximum rates of profit or maximum wage rates.

This completes the preliminaries. We can now discuss in general the problem of choice of technique raised initially in Section 2.3 in the context of a simple model. Given the specification of the technology, the problem of choice of technique is equivalent to that of determining the optimal economic lifetime of a machine.

\subsection{Negative ageing machine prices}

We consider the question of negative ageing machine prices in the context of the $(n+1)$-sector fixed capital model. Assume that all techniques are productive. Suppose that, at a feasible value of $r$, i.e. $r \in\left[0, R_{N}\right], p_{N}$ contains at least one negative component. As $r \leqslant R_{N}$, it follows that the negative component must refer to ageing machine prices. Let the first negative component of $p_{N}$ be $p_{n+h+1}$. In Theorem 6, we prove that technique $(h)$ supports a higher real wage than technique $(N)$ at the given value of $r$; consequently, $(h)$ is preferred to $(N)$. We first prove the Theorem before discussing its implications.

The proof is divided into two parts. We must, first of all, establish that the given value of $r$ is feasible for technique $(h)$, given $p_{n+h+1}<0$, that is, we must show that $r<R_{h}$. We can then apply Theorems 1-5 to technique $(h)$ as well as to $(N)$ to derive the result in the second part of the proof.

Theorem 6. $A$ "deleted" technique, say $(h)$, supports a higher real wage than $(N)$ if and only if $p_{N}$ has at least one negative component referring to ageing machines.

Proof. (i) Let $p_{n+h+1}$ be the first negative component of $p_{N}$ at $r \in\left[0, R_{N}\right]$. Take the first $(n+h)$ equations of (36),

$$
(1+r) p_{h} A_{h}+w_{N} l_{h}=p_{h} B_{h}+p_{n+h+1} e^{n+h}
$$

where $p_{h}=\left(p_{1}, \ldots, p_{n+1}, p_{n+2}, \ldots, p_{n+h}\right)>\mathbf{0}$ is a subvector of $p_{N} . A_{h}, l_{h}$ and $B_{h}$ are as defined in (81). $e^{n+h}$ is the $(n+h)$ th unit vector of order $(n+h)$. Rewrite (85) as

$$
p_{h}\left[B_{h}-(1+r) A_{h}\right] \geqslant w_{N} l_{h}>\mathbf{0}
$$

as $p_{n+h+1}<0$ by assumption. As $(h)$ is productive, it follows from Theorem 5 that

$$
\left[B_{h}-\left(1+R_{h}\right) A_{h}\right] x_{h}=\mathbf{0}
$$

where $R_{h}>0, x_{h}>0$. Post-multiplying (86) by $x_{h}>0$, premultiplying (87) by $p_{h}>0$ yields

$$
p_{h}\left[B_{h}-(1+r) A_{h}\right] x_{h}>0,
$$




$$
p_{h}\left[B_{h}-\left(1+R_{h}\right) A_{h}\right] x_{h}=0 .
$$

As $p_{h} A_{h} x_{h}>0$, it follows from (88) and (89) that

$$
R_{h}>r \text {. }
$$

So, if $(h)$ satisfies a productivity condition, $R_{h}>0$ and the given value of $r$, at which $p_{n+h+1}<0$, is feasible not only for $(N)$ but also for $(h)$.

(ii) Having established feasibility of $r$ for $(h)$, we can now apply Theorems 1-5 to $(h)$. Rewrite (85) as

$$
p_{h}\left[B_{h}-(1+r) A_{h}\right]=p_{n+h+1} e^{n+h}=w_{N} l_{h} .
$$

As $r$ is feasible for $(h)$, i.e. $r<R_{h}$, $\left[B_{h}-(1+r) A_{h}\right]^{-1}=C_{h}^{-1}$ exists. Equation (91) becomes

$$
p_{h}+p_{n+h+1} e^{n+h} C_{h}^{-1}=w_{N} l_{h} C_{h}^{-1} .
$$

Choose the $i$ th commodity as numeraire, $i=1, \ldots, n+1$. Post-multiplying (92) by $e^{i}, i=1, \ldots, n+1$, the $i$ th unit vector of order $(n+h)$ yields

$$
1+p_{n+h+1} e^{n+h} C_{h}^{-1} e^{i}=w_{N}^{(i)} l_{h} C_{h}^{-1} e^{i} .
$$

Applying Theorem 4 to $(h)$, we have

$$
w_{h}^{(i)}=1 / l_{h} C_{h}^{-1} e^{i}
$$

From Theorem 3 applied to $(h)$, we have

$$
\alpha_{n+h, i}=e^{n+h} C_{h}^{-1} e^{i}>0,
$$

where $\alpha_{n+h, i}$ is the $(n+h, i)$ element of $C_{h}^{-1}$; and $i$ can assume only the values $1, \ldots, n+1$. Using (94) and (95) in (93), we obtain

$$
p_{n+h+1} \alpha_{n+h, i}=w_{N}^{(i)} / w_{h}^{(i)}-1
$$

whence

$$
\operatorname{sign} p_{n+h+1}=\operatorname{sign}\left[w_{N}^{(i)}-w_{h}^{(i)}\right] .
$$

As $p_{n+h+1}<0$, it follows that $(h)$ can support a higher real wage than $(N)$. This establishes necessity.

To prove sufficiency, assume that $(h)$ supports a higher real wage than $(N)$ at a common feasible value of $r$; further assume that $p_{N}>0$. The argument above can be repeated except that now $p_{n+h+1}>0$. Equation (97) implies that

$$
w_{N}^{(i)}>w_{h}^{(i)}
$$

which contradicts the hypothesis. Hence, $p_{N}$ must contain at least one negative component. As $r$ is feasible for $(N)$, it follows that any negative component must refer to ageing machine prices. This completes the proof. 
There are a number of remarks to be made here.

REMARK 6. Theorem 6 does not permit us to infer that $(h)$ is actually chosen at the given value of $r$. It may happen that at least one of technique $(h)$ 's prices of production, obtained from (83) as

$$
p_{h}=w_{h} l_{h}\left[B_{h}-(1+r) A_{h}\right]^{-1}
$$

is negative. Then it follows from Theorem 6 that another technique, say $(f)$, obtained from $(h)$ by scrapping the ageing machine, is preferred to $(h)$. As there is a finite number of alternative techniques, it follows that the chosen techrịiuc ai the given value of $r$ is obtained after a finite number of steps.

REMARK 7. If $p_{h}$ has at least one negative component referring to ageing machines, there exists at the given value of $r$ another technique, say $(f)$, that is preferred to $(h)$. It follows from part (i) of the Theorem that $R_{f}>r$, just as $R_{h}>r$. In fact, $R_{h}$ places an upper limit on those values of $r$ at which $p_{n+h+1}<0$. For example, if $(h)$ is not productive, $R_{h}=0$ and $p_{n+h+1} \geqslant 0$. If $p_{n+h+1}<0$ at a feasible value of $r$, it follows that $(h)$ is a productive technique. As an extreme case, suppose that only technique $(N)$ is productive. It then follows that $p_{N}>\mathbf{0}$ for all $r \leqslant R_{N}$; for if $p_{N}$ had a negative component, there would be another technique that could support a higher real wage. This is impossible, as $(N)$ is the only productive technique.

REMARK 8. The significance of the Theorem lies in the fact that it enables us to determine the optimal economic lifetime of machinery by reference to the wage curves. Take the original technique $(N)$ and its associated productive deletions, $(1), \ldots,(N-1)$. Choose one marketable commodity (or a basket of marketable commodities) as numeraire. Construct the wage curve of each technique with respect to the numeraire, plotting all wage curves on the same diagram. Given a feasible value of $r$ (i.e. $0 \leqslant r \leqslant R_{\max }=\max _{h} R_{h}$ ), the optimal economic lifetime of the machine is given by that technique supporting the highest real wage.

\section{Conclusion}

Sraffa concluded in Section 84 of [8] that "a system which contained no other element of joint production besides what is implied in the presence of fixed capital would in general have an all-positive Standard Commodity, thus reproducing in this respect the simplicity of the system of single-product industries." ([8], page 75). We have formally proved this result in Theorem 5. In Theorems 1-4 and 6, we have established analogues for a fixed capital model of results familiar from the circulating capital model. 
In conclusion, it is appropriate to enquire into the generality of the results derived in Section 2. It has been demonstrated in another paper (Woods [10]) that the same methods as used above can be applied to a model where each sector employs an ageing machine. Consider a two-sector model which retains the single-product industries characteristic; suppose that the first sector produces iron, the second sector new machines. Suppose that the maximum physical lifetime of the new machine in the iron (respectively machine) sector is two (respectively three) periods. This is the simplest model in which fixed capital is employed in both sectors, with different lifetimes in the two sectors. Thus, it becomes necessary to distinguish between machines of the same type and age employed in different sectors.

Essentially the same procedure can be applied to this two-sector model as was applied to the $(n+1)$-sector model in Section 2. There are analogues of Theorems 1-6. In particular, the optimal economic lifetime of each machine can be determined. Note that the optimal economic lifetime of a particular machine is not determined in isolation; the problem is one in general equilibrium and can be resolved within a framework based on the same principles as in Section 2. Thus, it should not be thought that, by restricting ourselves to only one sector employing fixed capital, we are considering a partial equilibrium problem. The model constructed and analysed in Section 2 can be extended to accommodate the presence of fixed capital in any number of sectors, as long as the single-product industries postulate is retained.

\section{Acknowledgements}

A first draft of this paper was written while I was Visiting Fellow in the Department of Economics, University of Warwick, Coventry, England, during the summer term 1980-1. I would like to thank the Department for the facilities provided and the Arts and ESS Research Benefaction Fund, Trinity College, Dublin, for financial support. Non-incriminatory acknowledgements are due to the referees; I would particularly like to thank one of the referees for his, or her, exhaustive and constructive criticisms on each of the earlier versions of this paper. Finally, I thank Mrs. Lyons, Geography Department, Trinity College, for preparing the Figures.

\section{References}

[1] E. Burmeister and A. R. Dobell, Mathematical theories of economic growth (Macmillan, New York, 1970).

[2] M. Morishima, Equilibrium, stability and growth (Oxford University Press, Oxford, 1964).

[3] M. Morishima, The theory of economic growth (Oxford University Press, Oxford, 1969) 
[4] J. von Neumann, “A model of general economic equilibrium”, Rev. Econom. Stud. 13 (1945-6), $1-9$.

[5] P. Newman, "Production of commodities by means of commodities", Schweizer. Z. Volkswirtschaft und Statistik 98 (1962), 58-75.

[6] L. L. Pasinetti, Lectures on the theory of production, (Macmillan, London, 1977).

[7] L. L. Pasinetti (ed.), Essays on the theory of joint production (Macmillan, London, 1980).

[8] P. Sraffa, Production of commodities by means of commodities (Cambridge University Press, Cambridge, 1960).

[9] J. E. Woods, Mathematical economics (Longmans, London, 1978).

[10] J. E. Woods, "Matrices, the Perron-Frobenius theorem and the theory of fixed capital", paper presented at the European Meeting of the Econometric Society, Dublin, September, 1982. 